\title{
Research Article \\ Physicochemical and Functional Properties of Starches from Pachyrhizus erosus with Low Digestibility
}

\author{
Xiao-Dan Shi, Jing-Jing Huang, Jin-Zhi Han, Shao-Yun Wang* \\ College of Biological Science and Engineering, Fuzhou University, Fuzhou 350108, China
}

\section{ARTICLE INFO}

\section{Article History}

Received 04 May 2021

Accepted 22 June 2021

\section{Keywords}

Pachyrhizus erosus

starch

physicochemical properties

rheological properties

in vitro digestion

\begin{abstract}
The search for novel natural ingredients has been stimulated by the food and non-food industries, and roots of Pachyrhizus erosus (jicama) emerges as promising crops for industrial production due to its crispy and succulent flesh and high yield. Therefore, jicama starches from Fujian Province (SFJ) and Guangxi Province (SGX) were isolated and the microstructure, swelling power, crystallinity, thermal property, rheological property, and in vitro digestibility were determined. The starch granules of SFJ and SGX showed polyhedral, spherical or semi-spherical shapes with smooth nonporous surface. SFJ and SGX showed increasing solubility and swelling power with the increasing of temperature. By X-ray diffraction analysis, both had typical A-type crystallinity, but SFJ showed lower relative crystallinity (29.4\%) than SGX (35.4\%). SFJ with smaller granule sizes and lower relative crystallinity showed weaker thermal stability, lower viscosity, weaker strength of starch gels, and lower Resistant Starch (RS) content. The high contents of RS in SFJ (65.67\%) and SGX (70.37\%) suggested that jicama is promising low energy food for special purposes.
\end{abstract}

\section{HIGHLIGHTS}

- Jicama starches [Sample from Fujian Province (SFJ) and Sample from Guangxi Province (SGX)] were isolated and characterized by various techniques.

- SFJ and SGX showed similar shapes with smooth and nonporous surface.

- SFJ had smaller granule sizes and lower relative crystallinity.

- SFJ gels possessed weaker thermal stability, lower viscosity, and weaker strength.

- SGX had lower resistant starch content by in vitro digestion experiment.

(C) 2021 The Authors. Publishing services by Atlantis Press International B.V. This is an open access article distributed under the CC BY-NC 4.0 license (http://creativecommons.org/licenses/by-nc/4.0/).

\section{INTRODUCTION}

Starch is a kind of natural, renewable, and degradable carbohydrate, severing as basic metabolic energy provider. Different parts of plants, including seeds, roots, as well as stems, leaves, fruits and even pollens are common sources of starches [1,2]. Modern research on starches from various resources have demonstrated wide applications in food, plastic, adhesive, daily necessities, and pharmaceutical industries due to their distinguished gelling, film forming, and biodegradable properties [3-5]. Generally, starch is classified into Rapidly Digestible Starch (RDS), Slowly Digestible Starch (SDS), and Resistant Starch (RS) according to the rate of digestibility in the human body. Specifically, RDS is rapidly digested in the mouth and small intestine, and SDS is completely digested in the small intestine at a comparatively slow rate, while RS is used by microorganisms in the large intestine [6]. The digestibility properties of starch can reflect the nutritional values of food. At present, the major resources of starches in commercial market are maize,

"Corresponding author. Email: shywang@fzu.edu.cn

Peer review under responsibility of the International Association of Dietetic Nutrition and Safety

Data availability statement: The data that support the findings of this study are available from the corresponding author [SYW], upon reasonable request. cassava, potato, wheat, and rice, which have relatively low contents of RS [7]. With the growing market and industrial demands, exploration of new starches with different physicochemical, structural, and functional properties has been greatly motivated [8-10].

Pachyrhizus erosus root, also called jicama or yam bean, is a kind of vegetable with crispy and succulent flesh in white or light brown color. It can be eaten both in raw slices and cooked forms. This tropical/subtropical plant in legume family has been cultivated in Mexico since early times, and then being extensively imported to many other countries. It has high productivity and special sensory attributes, but it is underutilized at present [11]. A recent review reported that this un-exploited crop has potential applications in food industry, such as production of monosaccharides, oligosaccharides, high glucose syrups, flour, and wine, and being incorporated in yogurt or other milk products [12]. The tuberous jicama root contains about $80 \%$ water, $0.1 \%$ lipid, $0.5 \%$ ash, $1 \%$ protein, $15 \%$ carbohydrates, and $9 \%$ starch [13]. As a major component of the root, starch has been characterized in many studies [14-17]. Those starches from Pachyrhizus spp. were found to have small circular and polygonal shapes, and low pasting temperature [12]. At present, there are only a few investigations referring to the physicochemical and functional properties of jicama starches isolated that are widely cultivated in China. Since planting time and development stages 
has ever been found to influence the starch characteristics [18], it is reasonable to speculate that other cultivation conditions such as soil and cultivar may also play important roles. Thus, scientific data of starch from $P$. erosus cultivated in different regions in China are required to develop novel starch resources and improve the utilization value of this high-yield crop. Moreover, the digestibility or nutritional properties of jicama starches has not yet been clearly studied. Therefore, we isolated starches from jicama harvested in Fujian and Guangxi province. The microstructure, swelling power, thermal property, crystallinity, rheological property and in vitro digestibility were determined and compared. Our research may provide guidance for utilization of $P$. erosus and develop novel starch resources into the commercial markets.

\section{MATERIALS AND METHODS}

\subsection{Materials}

Fresh tubers of $P$. erosus were purchased from local markets in Fuzhou city (Fujian province, China) and Nanning city (Guangxi province, China), respectively. They were received at laboratory and processed immediately with wounded and unhealthy roots being discarded. Amyloglucosidase (70 U/mg) from Aspergillus niger amyloglucosidase and pancreatic $\alpha$-amylase (50 U/mg) were purchased from Sigma-Aldrich Inc. (St. Louis, MO, USA). All other chemicals and reagents were of analytical grade unless otherwise stated.

\subsection{Extraction of Jicama Starch}

The starch was isolated from the raw materials referring to a previous procedure [15]. Briefly, fresh roots were washed, peeled, chopped, and squeezed with distilled water. The mixture was drained with a \#200 mesh and the obtained solution was kept at $4^{\circ} \mathrm{C}$ for $12 \mathrm{~h}$ to precipitate the starch. The supernatant was removed by centrifugation $(4800 \mathrm{rpm}, 10 \mathrm{~min})$, and the precipitate was washed thoroughly with anhydrous ethanol and distilled water for several times to remove some contaminated compounds. The white powders after freeze-drying were named as SFJ and SGX.

\subsection{Structure Characterization}

\subsubsection{Optical microscopy}

The morphology of SFJ and SGX was first evaluated by optical microscope (OM) (Olypus DP73, Shinjuku, Tokyo, Japan). The starch sample was placed on a tube and mixed with 1-2 drops of water. A drop of the starch slurry was transferred onto the slide and then covered with a glass cover slip and the granule morphology was observed at $20 \times$ magnifications.

\subsubsection{Scanning electron microscopy (SEM)}

For the Scanning Electron Microscopy (SEM) observation, a FEI Nova NanoSEM system at high vacuum was used. The samples were fixed on an aluminum specimen holder with double graphite tape and then sputter-coated with gold particles. The measurement was done using a $5 \mathrm{kV}$ electron acceleration voltage. Diameters of starch granules were measured based on at least 30 starch granules on SEM micrographs $(5000 \times)$.

\subsubsection{Particle size distribution}

The size distribution of jicama starch granules was measured using a laser light scattering-based particle size analyzer (Malvern Instruments, Malvern, UK) according to the instructions supplied with the instrument. The starch was evenly dispersed in absolute ethanol at a concentration of $0.1 \%(\mathrm{w} / \mathrm{v})$ with magnetic agitation and sonication before test.

\subsubsection{Fourier transform infrared spectroscopy (FT-IR)}

Functional groups of the samples were determined by using a Nicolet 380 FT-IR spectrometer (Thermo Scientific, USA) within a range of wavenumbers from 4000 to $400 \mathrm{~cm}^{-1}$.

\subsubsection{Crystallinity of starch}

X-ray Diffraction (XRD) of starch was conducted using Empyrean PANalytical diffractometer (Netherlands) according to a modified method [19]. Prior to XRD analysis, the samples were freeze-dried and milled to pass through a 200 -mesh sieve. The scan was performed at $30 \mathrm{kV}$ and $10 \mathrm{~mA}$ for a $2 \theta$ range of $5-35^{\circ}$ with a step size of 0.01 . The relative crystallinity was calculated according to the report of Wang and Liu [20].

\subsection{Physicochemical Properties}

\subsubsection{Swelling power and solubility}

The starch sample $(0.2 \mathrm{~g})$ was finely dispersed into $10 \mathrm{~mL}$ distilled water and then the mixture was heated at $55,65,75,85$, and $95^{\circ} \mathrm{C}$ in a water bath for $60 \mathrm{~min}$. After heating, the samples were centrifuged at $3000 \mathrm{rpm}$ for $10 \mathrm{~min}$ (TDL-5-A, Shanghai Anting, China). The supernatant was dried at $105^{\circ} \mathrm{C}$, and the precipitate was weighed directly. The swelling power and solubility were calculated according to method reported by Karunaratne and Zhu [21].

\subsubsection{Transparency of starch pastes}

The transparency of starch pastes were measured using the previous method with a slight modification [22]. Briefly, the starch pastes were prepared as follows: an appropriate amount of starch was suspended in water to obtain a final $1 \%(\mathrm{w} / \mathrm{w})$ suspension. Each sample was cultivated in a water bath at $95 \pm 1{ }^{\circ} \mathrm{C}$ for $20 \mathrm{~min}$. During the heating process, the samples were refilled with water to replenish water loss due to evaporation, and starch paste was transferred to disposable spectrophotometric cuvettes. 
The measurements were performed at 5, 10, 30, and 60 min using a UV 1100 spectrophotometer (MAPADA Instruments, Shanghai, China) at a wavelength of $600 \mathrm{~nm}$ using water as control.

\subsubsection{Gelatinization properties}

Gelatinization properties were analyzed according to a published method with some modifications [23]. The onset temperature $\left(T_{o}\right)$, peak temperature $\left(T_{p}\right)$, conclusion temperature $\left(T_{c}\right)$, and gelatinization enthalpy $\left(\Delta H_{g}\right)$ of SFJ and SGX were monitored by Differential Scanning Calorimetry (DSC 214 Polyma, NETZSCHGerätebau $\mathrm{GmbH}$, Germany). In detail, accurately weighed sample was immersed in a 3:1 of liquid-solid ratio of deionized water. A drop of the mixed products was weighed into an aluminum pan and sealed quickly. It was equilibrated for $24 \mathrm{~h}$ at $4^{\circ} \mathrm{C}$ and then

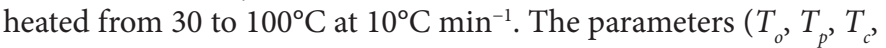
and $\Delta H_{g}$ ) were obtained from the DSC thermogram.

\subsubsection{Rheological properties}

The rheological properties of the starch samples were studied using a stress-controlled rheometer (MCR 302, Anton Paar, Austria) equipped with a $50 \mathrm{~mm}$ diameter parallel plate. A gap of $0.60 \mathrm{~mm}$ was used to perform all the rheological tests at $25^{\circ} \mathrm{C}$.

\subsubsection{Steady shear test}

Starch pastes at $1-5 \%$ (dry weight basis) of total solids were prepared by heating the starch dispersions in boiling water bath for $30 \mathrm{~min}$. Samples were equilibrated for about $2 \mathrm{~min}$ before test. In the shear rate sweep mode, the pastes were sheared from 0.1 to $1000 \mathrm{~s}^{-1}$ to describe the flow behavior.

\subsubsection{Dynamic oscillatory measurements}

A strain sweep measurement was performed at a constant frequency of $10 \mathrm{rad} \mathrm{s}^{-1}$ from $0.01 \%$ to $100 \%$ to determine the linear viscoelastic region. The dependence of loss modulus $\left(G^{\prime \prime}\right)$ and storage modulus $\left(G^{\prime}\right)$ on frequency were measured in frequency sweep mode. The strain was $2 \%$ and angular frequency from 1 to $100 \mathrm{rad} \mathrm{s}^{-1}$. The tests were done in the linear viscoelastic region. The creep experiment was conducted at constant stress of $0.8 \mathrm{~Pa}$, which was instantly applied and maintained for the duration of $180 \mathrm{~s}$. The creep compliance was calculated as the ratio of the total strain at aiven time to the constant stress [24].

\subsection{In vitro Digestibility}

The in vitro digestibility of SGX and SFJ was determined according to the methods of Englyst with some modifications [25,26]. Accurately, $200 \mathrm{mg}$ of starch samples were suspended in $15 \mathrm{~mL}$ of $0.2 \mathrm{M}$ sodium acetate buffer $(\mathrm{pH} 5.2)$ in $50 \mathrm{~mL}$ centrifuge tubes. Then, $5 \mathrm{~mL}$ of enzyme mixtures containing porcine pancreatic $\alpha$-amylase (300 $\left.\mathrm{U} \mathrm{mL}^{-1}\right)$ and amyloglucosidase $\left(20 \mathrm{U} \mathrm{mL}^{-1}\right)$, which were dissolved in $0.2 \mathrm{M}$ sodium acetate buffer ( $\mathrm{pH} 5.2$ ), were added into the starch suspension. The mixture was incubated at $37^{\circ} \mathrm{C}$ with stirring at $200 \mathrm{rpm}$ in a water bath. Aliquots $(0.5 \mathrm{~mL})$ of the hydrolysate were withdrawn at 20,40,60,80, 100, and $120 \mathrm{~min}$ and mixed with $0.5 \mathrm{~mL}$ of absolute ethanol to inactivate enzymes. After centrifuging at $3000 \mathrm{rpm}$ for $10 \mathrm{~min}$, the glucose contents of the hydrolysates were detected by a 3,5-dinitrosalicylic acid method. The glucose content (Gt) at intervals of different times were labeled as G20, G40, G60, G80, G100, and G120, respectively. Specifically, the hydrolysis rate (\%), RDS, SDS, and RS contents were calculated as follows:

$$
\begin{aligned}
& \text { Hydrolysis rate }(\%)=\frac{\mathrm{Gt}}{\mathrm{TS}} \times 0.9 \times 100 \\
& \operatorname{RDS}(\%)=\left[\frac{\mathrm{G} 20-\mathrm{FG}}{\mathrm{TS}}\right] \times 0.9 \times 100 \\
& \operatorname{SDS}(\%)=\left[\frac{\mathrm{G} 120-\mathrm{G} 20}{\mathrm{TS}}\right] \times 0.9 \times 100 \\
& \operatorname{RS}(\%)=\left[\frac{\mathrm{TS}-\mathrm{RDS}-\mathrm{SDS}}{\mathrm{TS}}\right] \times 100
\end{aligned}
$$

where G20 and G120 were the content of glucose after digestion for 20 and 120 min, respectively. FG was the free glucose content. TS was the total starch weight. 0.9 was the conversion factor of glucose to starch.

\section{RESULTS AND DISCUSSION}

\subsection{Structure Characterization of Starch}

\subsubsection{Morphology and particle size of starch granules}

As described above, fresh tuber of $P$. erosus was subjected to washing, slicing, and squeezing, and precipitating. Two kinds of starch samples (SFJ and SGX) were recovered from Fujian and Guangxi province, respectively. The yields of SFJ and SGX by three repeating experiments were $2.20 \pm 0.01 \%$ and $1.09 \pm 0.10 \%$, respectively. The starch morphology is considered important due to its relationship with amylose content, water absorption capability, and solubility. Figure 1 shows the micrograph of detected starches. In Figure 1a and 1b, the starches granules of SFJ and SGX observed by OM showed polyhedral, spherical or semi-spherical shapes with different sizes. More clearly, SEM images (Figure 1c and 1d) shows that those starch granules performed smooth and nonporous surface. By measuring the sizes of at least 30 unbroken particles in Figure 1c and 1d, the diameters of particles of SFJ and SGX varied from 3 to $9 \mu \mathrm{m}$, indicating that jicama starches belong to B-type starch according to the previous reports [27,28]. Averagely, SFJ $(5.2 \pm 1.5 \mu \mathrm{m})$ had more granules in smaller size than SGX $(5.4 \pm 1.6 \mu \mathrm{m})$. This result was close to the granule sizes determined by laser light scattering particle size analyzer, which was $3.16 \pm 1.40 \mu \mathrm{m}$ for SFJ and $4.41 \pm 1.66 \mu \mathrm{m}$ for SGX. The morphology of starch observed in our study was consistent with that of starch from isolated Jicama grown in Mexico [15]. The starches 

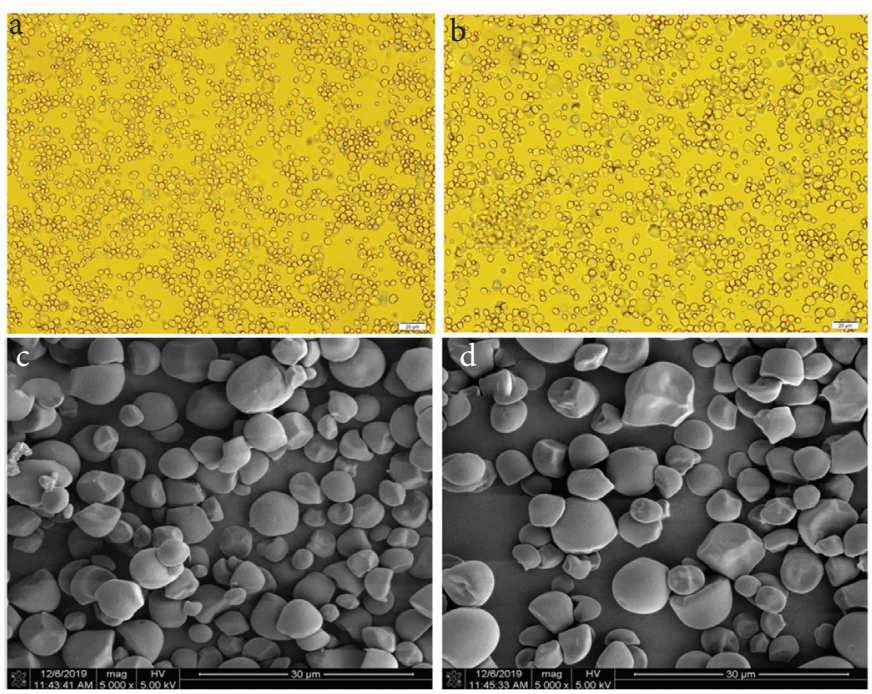

Figure 1 Micrographs of starch granules isolated from P. erosus cultivated in Fujian province (SFJ) and Guangxi province (SGX), respectively.

(a) SFJ observed in optical microscope at 20x; (b) SGX observed in optical microscope at 20×; (c) SFJ observed in Scanning Electron Microscope (SEM) at 5000×; (d) SGX observed in SEM at 5000×.

also had similar shape with granules of maize starches [24], and their sizes were similar with those of young bamboo culm starches [29].

\subsubsection{FT-IR spectra of SFJ and SGX}

The FT-IR spectra of SFJ and SGX are presented in Figure 2 with the labeled wavenumbers of the main peaks. The two samples possessed similar chemical structure according to the FT-IR spectra. In detail, the absorption bands at around 3300-3600, 2900, 1150 (700-900), and 1000-1100 (1648) $\mathrm{cm}^{-1}$ in the spectra indicated the presence of $\mathrm{OH}, \mathrm{C}-\mathrm{H}, \mathrm{C}-\mathrm{O}-\mathrm{C}$, and $\mathrm{C}-\mathrm{O}$ functional groups, respectively $[30,31]$. Furthermore, a group of absorbance peaks at around $1300-1500 \mathrm{~cm}^{-1}$ showed the existence of $\mathrm{C}-\mathrm{H}$ in $-\mathrm{CH}_{2} \mathrm{OH}$ moiety. The uncommon peak at $2362 \mathrm{~cm}^{-1}$ was probably derived from $\mathrm{CO}_{2}$ in the air. The above data of SFJ and SGX were consistent with that of corn, cassava, and potato starches [30].

\subsubsection{Relative crystallinity by XRD analysis}

The crystallinity of starch is a key index of starch, which is jointly determined by many factors, such as the amylopectin content, chain length, and distribution of amylopectin, crystal size, the orientation and extent of interaction between double helices [32]. According to the XRD patterns in Figure 3a, starch granules of SFJ and SGX showed strong diffraction peaks at $15^{\circ}, 17^{\circ}, 18^{\circ}$, and $23^{\circ}$ (Figure $3 \mathrm{~d}$ ), belonging to a typical A-type crystallinity. SFJ showed lower relative crystallinity (29.4\%) than SGX (35.4\%), indicating that SGX had higher thermal stability due to the fact that higher degree of crystallinity results in higher gelatinization temperature [30].

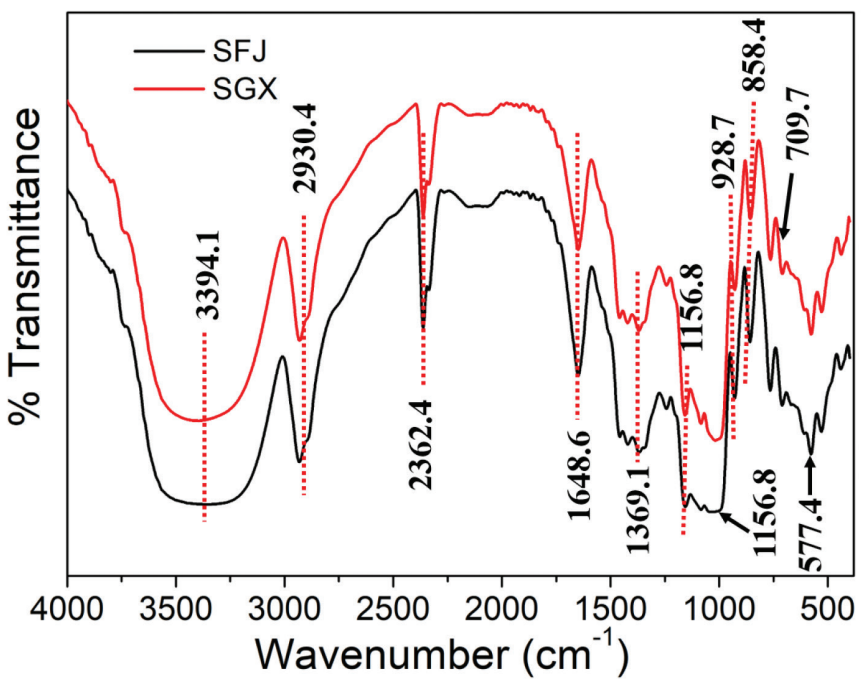

Figure 2 FT-IR spectroscopy of starches granules respectively isolated from P. erosus cultivated in Fujian province (SFJ) and Guangxi province (SGX).

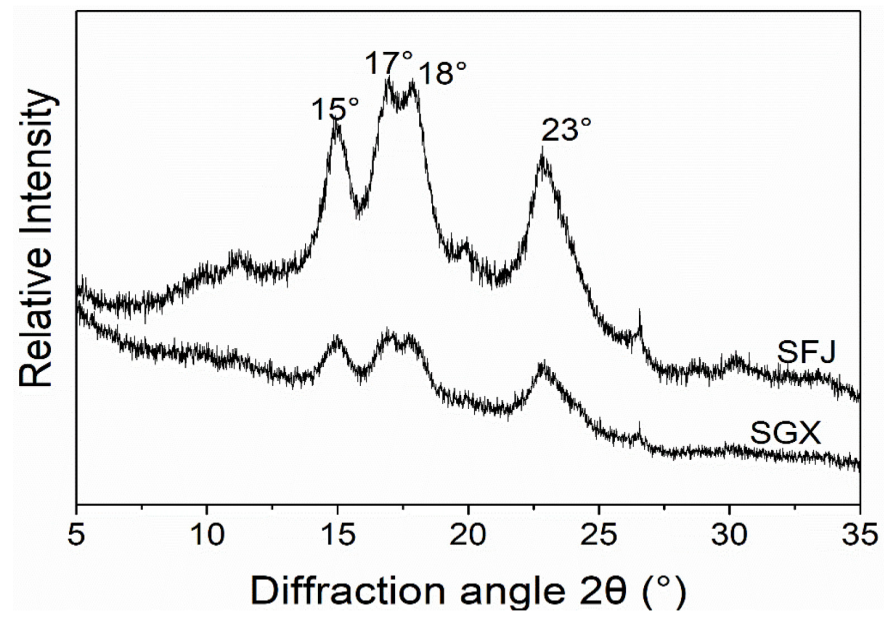

Figure 3 XRD curves of starches from $P$. erosus cultivated in Fujian (SFJ) and Guangxi (SGX).

\subsection{Physicochemical Properties of SFJ and SGX}

\subsubsection{Swelling power, solubility, and transparency}

Swelling power of starch reflects its hydration ability, during which the expansion of starch granules can promote the attack of the internal structure by digestive enzymes, resulting in increasing rate of starch hydrolysis. Figure $4 \mathrm{a}$ and $4 \mathrm{~b}$ shows the swelling power and solubility of SFJ and SGX at various temperatures. Obviously, the starch particles swelled to only a small extent at $55-65^{\circ} \mathrm{C}$; however, they expended quickly when the temperature was increased to $65-95^{\circ} \mathrm{C}$. The swelling pattern in our investigation were similar with those of sweet potato starch [33], but different from those of corn starches reported before [31]. The decreasing trend of 

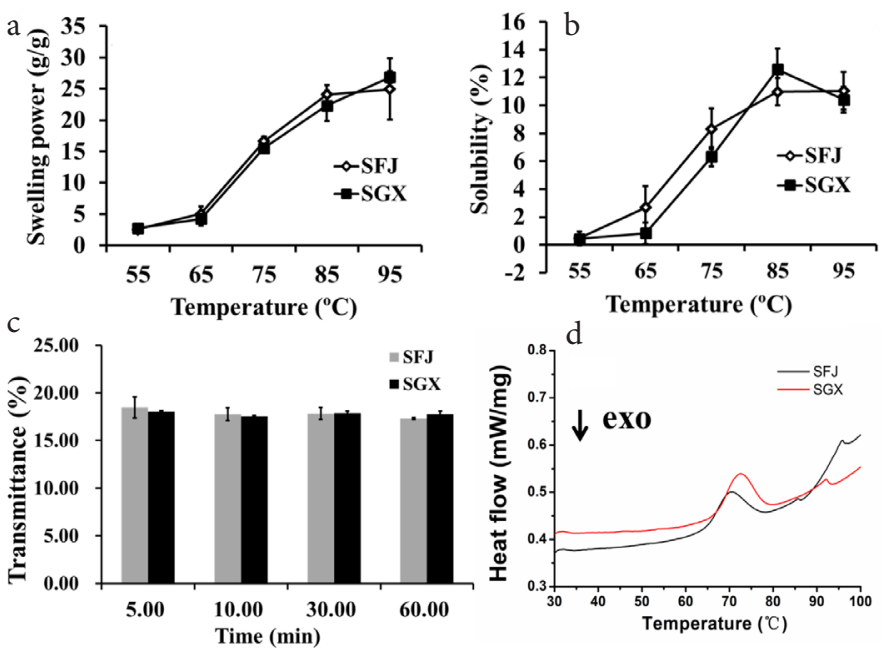

Figure 4 Swelling power (a), solubility (b), transparency (c), and DSC curves (d) of starches from P. erosus cultivated in Fujian (SFJ) and Guangxi (SGX).

swelling of SFJ at $95^{\circ} \mathrm{C}$ may be due to the increasing activity of $\alpha$-amylase at high temperature or the leaching out of cloudy solids caused by heating [33]. Though the swelling power and solubility of SFJ and SGX had similar increasing trend with the temperature ramping from 55 to $95^{\circ} \mathrm{C}$, there were some differences between the two starch samples. Firstly, at lower temperature $\left(55-75^{\circ} \mathrm{C}\right)$, the solubility of SFJ was a little higher than that of SGX, which may be attributed to the smaller size of its granules [34]. Secondly, SGX hold stronger swelling power than $\mathrm{SFJ}$ at $95^{\circ} \mathrm{C}$; however, it showed lower solubility.

Transparency provides information of the behavior of starch paste when the light passes through it. Its value depends on granule size, swelling capabilities, amylose content, and amylose/amylopectin ratio. The transparency of $1 \%(\mathrm{~m} / \mathrm{v})$ starch pastes in Figure $4 \mathrm{c}$ was about $18 \%$ and showed no big change in $60 \mathrm{~min}$. The value was close to that of chestnut starch but much lower than the transparency value of potato starch [35].

\subsubsection{Thermal property by DSC}

Thermal property related crucial parameters $\left(T_{o}, T_{p}, T_{c}\right.$, and $\left.\Delta H_{g}\right)$ derived from DSC diagram (Figure $4 \mathrm{~d}$ ) were summarized in Table 1. The SFJ showed lower gelatinization temperatures than SGX, which could be attributed to its relatively smaller starch granules and lower crystallinity [30]. According to Abdullah et al., the gelatinization temperature is a key parameter in the bioplastic production. If it is lower than $60^{\circ} \mathrm{C}$, the starch slurry might be gelatinized in the drying process; if too high, the production process causes high energy cost [30]. Therefore, the starch from P. erosus may be potential to be used in the bioplastic production.

The $\Delta H_{g}$ in our test is around $3.0 \mathrm{~J} \mathrm{~g}^{-1}$, which is greatly lower than the values of common starches from various sources, such as potato, Tapioca, waxy corn, sweet potato [33], but close to the data of starches from young bamboo culm of Dendrocalamus asper [29]. Given that sample with more B-type starch granules are associated with low $\Delta H_{g}$ and relative crystallinity, the low $\Delta \mathrm{H}_{g}$ of starch from P. erosus may be related to their B-type starch granules [34].

\subsection{Rheological Properties}

\subsubsection{Steady shear rheological properties}

The viscosity curves of SFJ and SGX gels from $1 \%(\mathrm{w} / \mathrm{v})$ to $5 \%$ $(\mathrm{w} / \mathrm{v})$ by steady shear experiment at $25^{\circ} \mathrm{C}$ were in Figure $5 \mathrm{a}$ and $5 \mathrm{~b}$, respectively. The apparent viscosities of SFJ and SGX gels presented shear-thinning behaviors, which belonged to the typical pseudo-plastic fluids. The comparative curves of SFJ and SGX at $5 \%(\mathrm{w} / \mathrm{v})$ in Figure $5 \mathrm{c}$ obviously showed that SFJ had lower viscosity than SGX.

\subsubsection{Dynamic rheological properties}

The storage modulus $\left(G^{\prime}\right)$ and loss modulus $\left(G^{\prime \prime}\right)$ for $5 \%(\mathrm{w} / \mathrm{v})$ SFJ and SGX at $25^{\circ} \mathrm{C}$ and a strain of $2.0 \%$ were presented in Figure $5 \mathrm{~d}$. The magnitudes of $G^{\prime}$ and $G^{\prime \prime}$ were frequency dependent and there was no crossover between these two moduli throughout $0.1-100$ $\mathrm{rad} \mathrm{s}^{-1}$. In addition, the magnitudes of $G^{\prime}$ and $G^{\prime \prime}$ increased with the increasing angular frequency and $G^{\prime}$ value was always much higher than $G^{\prime \prime}$ value. These results suggested that both samples probably showed a typically rheological behavior of weak gels [36]. Apparently, both moduli of SGX were much higher than those of SFJ. Therefore, the SGX gel tended to have stronger gel strength than SFJ.

\subsubsection{Creep experiment}

The creep curves of SFJ and SGX at concentrations of $1-5 \%(\mathrm{w} / \mathrm{v})$ were presented in Figure 5. In the creep experiment, a constant stress $(0.8 \mathrm{~Pa})$ was applied to the sample and the compliance was monitored over time. Generally, a non-linear response to stress indicated normal behavior of viscoelastic materials that combined both elastic and viscous components [24]. The compliance value is an indicator of strength of gels. A low compliance value corresponded to a strong system with low deformability, whereas a high compliance corresponded to a weak system with high deformability [24]. All creeping curves of SFJ and SGX rapidly reached the maximum compliance values, which decreased with the increase of mass concentrations. This result meant that the higher the starch concentration was, the stronger the gel was. By comparing the two starch samples, SFJ exhibited higher compliance values during the creep test at the same concentration. Therefore, the strength of the starch gels of SFJ was weaker than SGX, agreeing with the result of frequency sweep.

Table 1 DSC parameters and contents of RDS, SDS, and RS of starches from P. erosus cultivated in Fujian (SFJ) and Guangxi (SGX)

\begin{tabular}{lcr}
\hline Samples & SFJ & \multicolumn{1}{c}{ SGX } \\
\hline DSC parameters of gelatinization & & \\
$T_{o}\left({ }^{\circ} \mathrm{C}\right)$ & $61.7 \pm 0.1$ & $65.2 \pm 0.5$ \\
$T_{p}\left({ }^{\circ} \mathrm{C}\right)$ & $70.7 \pm 0.1$ & $73.3 \pm 0.5$ \\
$T_{c}\left({ }^{\circ} \mathrm{C}\right)$ & $79.3 \pm 0.0$ & $80.5 \pm 0.6$ \\
$\Delta H_{g}\left(\mathrm{~J} \mathrm{~g}^{-1}\right)$ & $3.1 \pm 0.2$ & $2.9 \pm 0.3$ \\
Contents of RDS, SDS, and RS & & \\
RDS (\%) & $16.22 \pm 0.49$ & $11.42 \pm 0.62$ \\
SDS $(\%)$ & $18.45 \pm 0.98$ & $18.02 \pm 1.14$ \\
RS (\%) & $65.67 \pm 0.88$ & $70.37 \pm 1.82$ \\
\hline
\end{tabular}


a
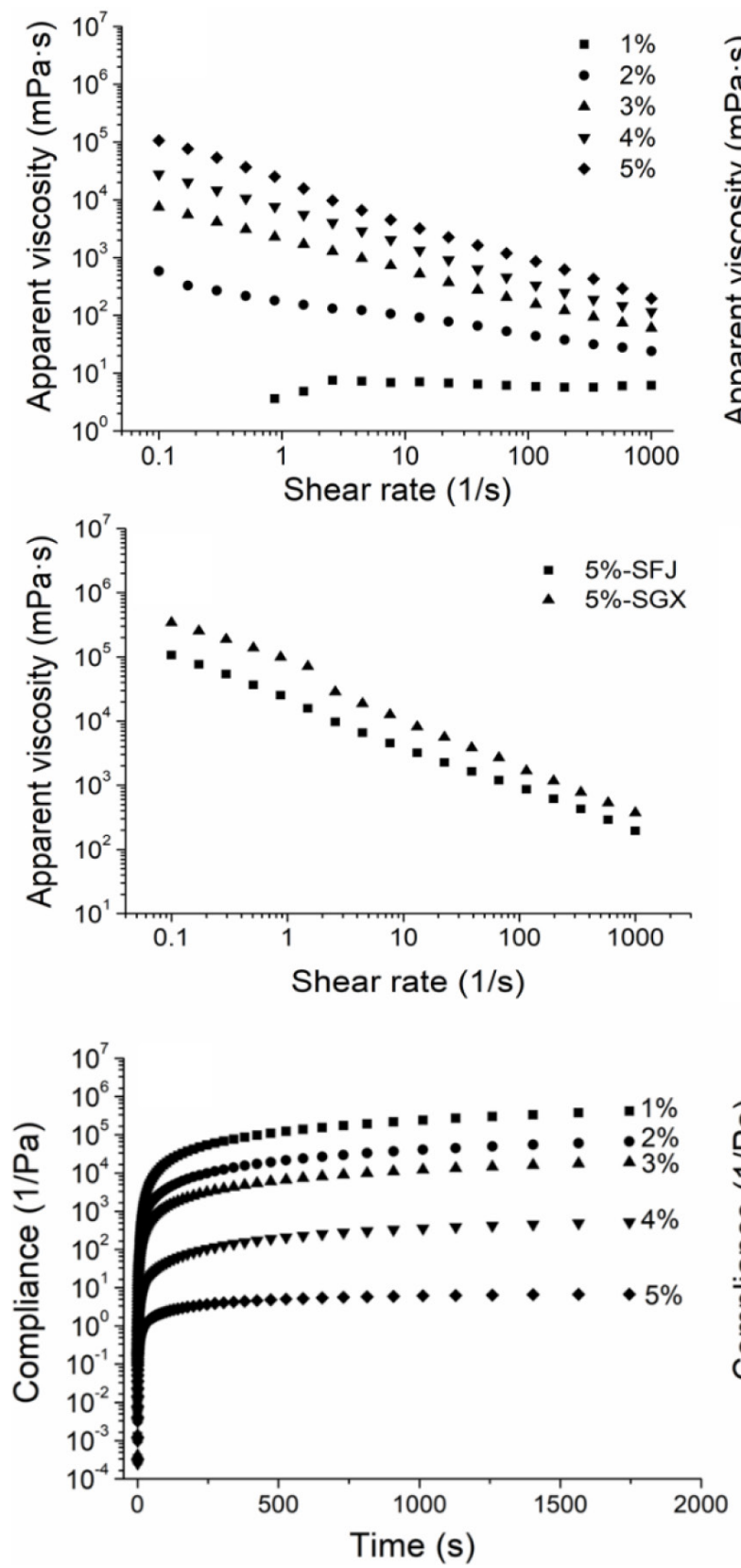
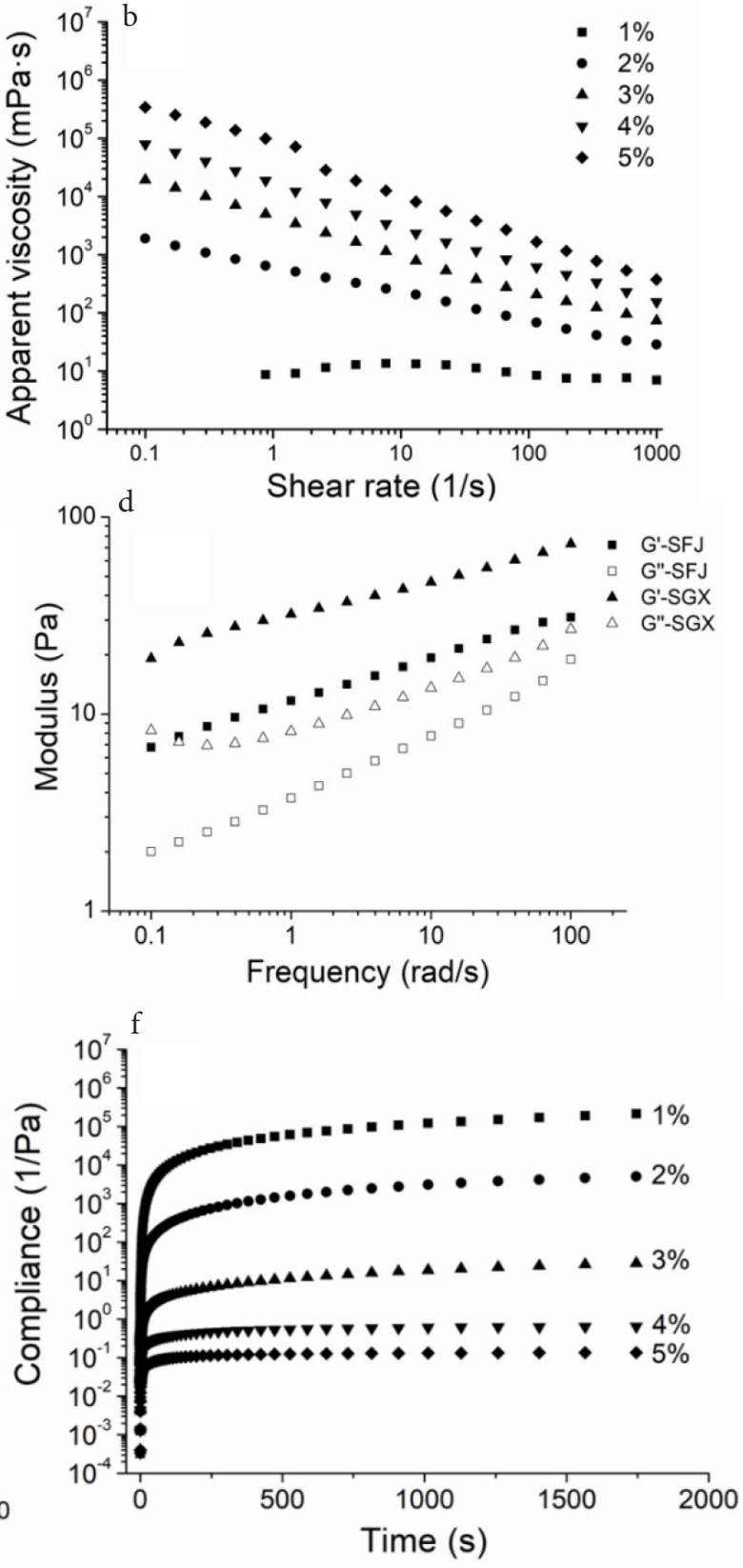

Figure 5 Rheological properties of SFJ and SGX from P. erosus. (a) Apparent viscosity of SFJ under different concentrations; (b) apparent viscosity of SFJ under different concentrations; (c) comparison of shear rate curves of 5\% (w/v) SFJ and SGX; (d) storage modulus and loss modulus of 5\% (w/v) SFJ and SGX by frequency sweep experiment; (e) and (f) creep curves of SFJ SGX under different concentrations, respectively.

\subsection{In vitro Digestion Properties}

Contents of RDS, SDS, and RS could be indicator of the nutritional value of starch food such as the postprandial glucose and insulin response. Currently, RDS is the major content of most common starches. For example, there were about $70 \%$ of RDS in corn starch [37], 96\% in barley starch [38], and 76-80\% in cassava starch [39]. As displayed in Table 1, SFJ and SGX had extremely high contents of RS (65-70\%) and low contents of RDS (11-16\%), indicating that SFJ and SGX were resistant to digestion. Similar contents of RS of starches have been reported from Chinese chestnuts (67-85\%) [6,40] and naked oat $(68-76 \%)$ [41]. However, the SDS contents of SFJ and SGX were lower than those from chestnuts (5-20\%), but higher than oats $(\sim 10 \%)$. The hydrolysis rates of SFJ and SGX by porcine pancreatic $\alpha$-amylase and amyloglucosidase at different time intervals were shown in Figure 6. Different from other starches such as corn starch, the hydrolysis curves of SFJ and SGX did not fit to the first-order equation as described previously [37], which may be attributed to their chemical structures.

\section{CONCLUSION}

In this study, starches (SFJ and SGX) from jicama cultivated in Fujian and Guangxi province in China were extracted, and they presented characteristics of small diameter $(3-9 \mu \mathrm{m})$ and A-type crystallinity. Thermal and rheological analysis suggested that SFJ had some distinctions in gelatinization temperature, viscosity, starch gel strength, and compliance values with those of SGX, which may be 


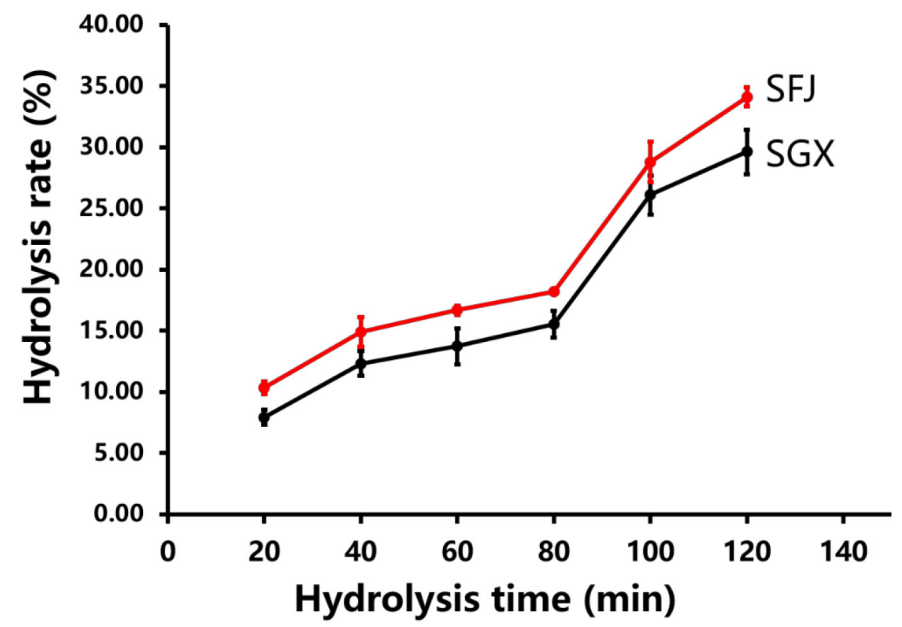

Figure 6 Kinetics of enzymatic hydrolysis of SFJ and SGX from P. erosus.

attributed to its smaller granule size and lower crystallinities. For in vitro digestion, both SFJ and SGX had low contents of RDS, relatively low contents of SDS, and high contents of RS. The obtained results in this investigation confirmed that the physicochemical and functional properties of starches might be closely related to the chemical structure of starch. With the increasing demand for novel starch products and resources, jicama starches may be an alternative source of starches to those traditionally used ones. To develop starch from jicama as a new ingredient for industrial applications, more research is required to explore the gelatinization kinetics, retrogradation kinetics, and structure modifications in the future.

\section{CONFLICTS OF INTEREST}

The authors declare that they have no conflicts of interest.

\section{AUTHORS' CONTRIBUTION}

XDS contributed in conceptualization, formal analysis, investigation, writing-original draft, software. JJH contributed in resources, investigation, formal analysis. JZH contributed in conceptualization, visualization, writing-review \& editing. SYW contributed in conceptualization, supervision, visualization, writing-review \& editing, validation.

\section{ACKNOWLEDGMENTS}

This work was supported by Natural Science Foundation of China (U1905202) and Fuzhou University Testing Fund of Precious Apparatus (2021T016).

\section{REFERENCES}

[1] Ashogbon AO, Akintayo ET. Recent trend in the physical and chemical modification of starches from different botanical sources: a review. Starch-Stärke 2014;66:41-57.

[2] Karim AA, Tie APL, Manan DMA, Zaidul ISM. Starch from the sago (Metroxylon sagu) palm tree-properties, prospects, and challenges as a new industrial source for food and other uses. Compr Rev Food Sci Food Saf 2008;7:215-28.

[3] Han Z, Shi R, Sun DW. Effects of novel physical processing techniques on the multi-structures of starch. Trends Food Sci Technol 2020;97:126-35.

[4] Kumari S, Yadav BS, Yadav RB. Synthesis and modification approaches for starch nanoparticles for their emerging food industrial applications: a review. Food Res Int 2020;128:108765.

[5] Miao M, Jiang B, Cui SW, Zhang T, Jin Z. Slowly digestible starch—a review. Crit Rev Food Sci Nutr 2015;55:1642-57.

[6] Wang Y, Zhao J, Wu Y, Wang M, Ouyang J. Processing of air-dried chestnut and physicochemical properties of its starch with low digestibility. Food Hydrocolloids 2020;108:106051.

[7] Waterschoot J, Gomand SV, Fierens E, Delcour JA. Production, structure, physicochemical and functional properties of maize, cassava, wheat, potato and rice starches. Starch-Starke 2015;67:14-29.

[8] Kringel DH, Dias ARG, da Rosa Zavareze E, Gandra EA. Fruit wastes as promising sources of starch: extraction, properties, and applications. Starch-Starke 2020;72:1900200.

[9] Przetaczek-Rożnowska I, Fortuna T. Effect of conditions of modification on thermal and rheological properties of phosphorylated pumpkin starch. Int J Biol Macromol 2017;104:339-44.

[10] Santacruz S, Ruales J, Eliasson AC. Three under-utilised sources of starch from the Andean region in Ecuador. Part II. Rheological characterisation. Carbohydr Polym 2003;51:85-92.

[11] Ramos-de-la-Peña AM, Renard CMGC, Wicker L, Montañez J, de la Luz Reyes-Vega M, Contreras-Esquivel JC. Optimization of the liquefaction and saccharification of structural polysaccharides of jicama (Pachyrhizus erosus L.) tissue by enzymatic pulping. LWT - Food Sci Technol 2012;46:232-8.

[12] Ramos-de-la-Peña AM, Renard CMGC, Wicker L, ContrerasEsquivel JC. Advances and perspectives of Pachyrhizus spp. in food science and biotechnology. Trends Food Sci Technol 2013;29:44-54.

[13] Noman ASM, Hoque MA, Haque MM, Pervin F, Karim MR. Nutritional and anti-nutritional components in Pachyrhizus erosus L. tuber. Food Chem 2007;102:1112-18.

[14] Amaya-Llano SL, Martínez-Bustos F, Alegría ALM, de Jesús Zazueta-Morales J. Comparative studies on some physico-chemical, thermal, morphological, and pasting properties of acid-thinned jicama and maize starches. Food Bioprocess Technol 2011;4:48-60.

[15] Contreras-Jiménez B, Vázquez-Contreras G, de los Ángeles Cornejo-Villegas M, del Real-López A, Rodríguez-García ME. Structural, morphological, chemical, vibrational, pasting, rheological, and thermal characterization of isolated jicama (Pachyrhizus spp.) starch and jicama starch added with $\mathrm{Ca}(\mathrm{OH})_{2}$. Food Chem 2019;283:83-91.

[16] Martínez-Bustos F, López-Soto M, Zazueta-Morales JJ, MoralesSánchez E. Preparation and properties of pre-gelatinized cassava (Manihot esculenta. Crantz) and jícama (Pachyrhizus erosus) starches using ohmic heating. Agrociencia 2005;39:275-83.

[17] Mélo EA, Stamford TLM, Silva MPC, Krieger N, Stamford NP. Functional properties of yam bean (Pachyrhizus erosus) starch. Bioresour Technol 2003;89:103-6.

[18] Leonel M, Ferrari TB, Sarmento SBS, de Oliveira MA. Planting time, developmental stages and characteristics of roots and starch of Pachyrhizus ahipa. Sci Agric 2005;62:528-33.

[19] Oyeyinka SA, Singh S, Ma Y, Amonsou EO. Effect of highpressure homogenization on structural, thermal and rheological properties of bambara starch complexed with different fatty acids. RSC Adv 2016;6:80174-80. 
[20] Wang W, Liu J. Efficient extraction, antioxidant activities and anti-inflammation of polysaccharides from Notopterygium franchetii Boiss. Carbohydr Polym 2020;248:116783.

[21] Karunaratne R, Zhu F. Physicochemical interactions of maize starch with ferulic acid. Food Chem 2016;199:372-9.

[22] Berski W, Ziobro R, Witczak M, Gambuś H. The retrogradation kinetics of starches of different botanical origin in the presence of glucose syrup. Int J Biol Macromol 2018;114: 1288-94.

[23] Shen X, Shang W, Strappe P, Chen L, Li X, Zhou Z, et al. Manipulation of the internal structure of high amylose maize starch by high pressure treatment and its diverse influence on digestion. Food Hydrocolloids 2018;77:40-8.

[24] Chen L, Tian Y, Bai Y, Wang J, Jiao A, Jin Z. Effect of frying on the pasting and rheological properties of normal maize starch. Food Hydrocolloids 2018;77:85-95.

[25] Ding Y, Xiao Y, Ouyang Q, Luo F, Lin Q. Modulating the in vitro digestibility of chemically modified starch ingredient by a non-thermal processing technology of ultrasonic treatment. Ultrason Sonochem 2021;70:105350.

[26] Englyst HN, Kingman SM, Cummings JH. Classification and measurement of nutritionally important starch fractions. Eur J Clin Nutr 1992;46:S33-S50.

[27] Cao H, Yan X, Chen G, Zhou J, Li X, Ma W, et al. Comparative proteome analysis of A- and B-type starch granule-associated proteins in bread wheat (Triticum aestivum L.) and Aegilops crassa. J Proteom 2015;112:95-112.

[28] Peng M, Gao M, Abdel-Aal E-SM, Hucl P, Chibbar RN. Separation and characterization of $\mathrm{A}$-and $\mathrm{B}$-type starch granules in wheat endosperm. Cereal Chem 1999;76:375-9.

[29] Felisberto MHF, Beraldo AL, Costa MS, Boas FV, Franco CML, Clerici MTPS. Characterization of young bamboo culm starch from Dendrocalamus asper. Food Res Int 2019;124:222-9.

[30] Abdullah AHD, Chalimah S, Primadona I, Hanantyo MHG. Physical and chemical properties of corn, cassava, and potato starchs. IOP Conference Series: Earth and Environmental Science. Jakarta, Indonesia: IOP Publishing; 2018, p. 012003.
[31] Lopez-Silva M, Bello-Perez LA, Agama-Acevedo E, AlvarezRamirez J. Effect of amylose content in morphological, functional and emulsification properties of OSA modified corn starch. Food Hydrocolloids 2019;97:105212.

[32] Rafiq SI, Jan K, Singh S, Saxena DC. Physicochemical, pasting, rheological, thermal and morphological properties of horse chestnut starch. J Food Sci Technol 2015;52:5651-60.

[33] Li JY, Yeh AI. Relationships between thermal, rheological characteristics and swelling power for various starches. J Food Eng 2001;50:141-8.

[34] Cao X, Tong J, Ding M, Wang K, Wang L, Cheng D, et al. Physicochemical properties of starch in relation to rheological properties of wheat dough (Triticum aestivum L.). Food Chem 2019;297:125000.

[35] Chang H, Zhou JH, Lan YP, Liu SQ, Zhai JJ. Comparative study on physical properties of different starch paste. Food Res Dev 2010;31:55-8.

[36] Ikeda S, Nishinari K. "Weak gel"-type rheological properties of aqueous dispersions of nonaggregated $K$-carrageenan helices. J Agric Food Chem 2001;49:4436-41.

[37] Ning Y, Cui B, Yuan C, Zou Y, Liu W, Pan Y. Effects of konjac glucomannan on the rheological, microstructure and digestibility properties of debranched corn starch. Food Hydrocolloids 2020;100:105342.

[38] Liu K, Zhang B, Chen L, Li X, Zheng B. Hierarchical structure and physicochemical properties of highland barley starch following heat moisture treatment. Food Chem 2019;271:102-8.

[39] Gourilekshmi SS, Jyothi AN, Sreekumar J. Effect of cultivar difference and root growth stage on the thermal, rheological, and in vitro digestibility properties of cassava starch. Starch-Stärke 2021;73:2000157.

[40] Liu C, Wang S, Chang X, Wang S. Structural and functional properties of starches from Chinese chestnuts. Food Hydrocolloids 2015;43:568-76.

[41] Tang M, Wang L, Cheng X, Wu Y, Ouyang J. Non-starch constituents influence the in vitro digestibility of naked oat (Avena nuda L.) starch. Food Chem 2019;297:124953. 